\title{
LG CNS Digital Transformation Using Robotic Process Automation
}

\author{
Jung Ho Lee ${ }^{1}$, Eunyoung Lim² ${ }^{2}$ Namho Chung ${ }^{1}$ \\ ${ }^{1}$ Kyung Hee University, Seoul, Korea \\ ${ }^{2}$ LG CNS Al Business Unit, Seoul, Korea
}

Objectives: Robotic process automation (RPA) has emerged as a trend in various industries due to the increasing interest in digital transformation and innovations. RPA has been actively applied in the financial and service industries, and the ability to integrate artificial intelligence (AI) technology has prompted firms in many industries to adopt RPA to increase the efficiency of their business operations. This study aims to find out how companies adopt RPA based on LG CNS' RPA application cases.

Methods: The method of collective case studies with multiple case was used. To this end, this study analyzed and organized multiple cases exemplifying the applications of RPA in LG CNS for various purposes, such as the verification of outside information from an unassociated system, simple information verification, system input tasks, and data reconciliation.

Results: Several considerations should be kept in mind for the functional implementation of AI-based RPA. Research is needed on the characteristics of specific types of applications, and an optimized RPA solution requires careful consideration of which processes will be automated by AI and how best to do so.

Conclusions: RPA automates simple repetitive tasks, standardizing business processes and helping to increase the efficiency of administrative processes. Therefore, RPA should be validated for use in administrative tasks at public institutions. However, there are limits and points to consider from the standpoint of application processes, legislative issues, and governance. For these reasons, there is a need for more research and case studies on RPA technology and related industries before it can be deployed more widely.

Key Words: LG CNS, Robotic Process Automation, Artificial Intelligence, Intelligence Process Automation, Digital Transformation

\section{Introduction}

In this time of rapid changes, when agility is an important factor for industries and organizations to remain competitive, tech-

Received: Nov 28, 2021 Revised: Dec 30, 2021 Accepted: Jan 3, 2022 Corresponding author: Namho Chung

Smart Tourism Education Platform, Kyung Hee University, 26 Kyungheedaero, Dongdaemun-gu, Seoul 02447, Korea

Tel: +82-2-961-2353, E-mail: nhchung@khu.ac.kr

This is an Open Access article distributed under the terms of the Creative Commons Attribution Non-Commercial License (http://creativecommons.org/licenses/ by-nc/4.0/) which permits unrestricted non-commercial use, distribution, and reproduction in any medium, provided the original work is properly cited.

Copyright $\odot 2022$ Korean Association for Business Communication. nological adaptation is crucial. The emergence of technological innovations and advances, such as web 2.0, smartphones, and artificial intelligence (AI), disrupted many industries and led to major changes to business strategies (Devarajan, 2018; Syed et al., 2020). Many forms of digital transformation are under development, and this constant evolution in information technology (IT) is leading businesses to embrace the digitalization of processes and systems (Anagnoste, 2017). In particular, with advances in cognitive analytics, the interest in robotic process automation (RPA) has dramatically increased. According to the Gartner report, RPA is the fastest-growing software subsegment, with year-over-year growth of more than 63\% in 2018.

The generalization of digitalization, IT adoption, and com- 
petition in the Fourth Industrial Revolution era caused industries and companies to pay closer attention to automation opportunities and initiatives (Marengo, 2019). According to a Deloitte survey, over $78 \%$ of global corporations are planning on additional investment in RPA due to their high satisfaction with its effects on compliance, productivity in terms of quality/ accuracy, flexibility (with a satisfaction rate of over $90 \%$ ), cost reduction, and implementation speed (with a satisfaction rate of over 60\%). Currently, corporations that are global leaders in RPA solutions, such as Automation Anywhere, Uipath, and Blue Prism, are promoting the RPA market by developing closely interlinked, well-developed partner ecosystems, providing business process outsourcing (BPO) consulting services, and other strategies. Globally leading manufacturing corporations are also situationally adapting various forms of RPA services. Oracle and ServiceNow have constructed optimized models for individual IT infrastructure environments. In contrast, Syntel, Tata Consulting, and Xerox are providing BPO-based RPA services. Thoughtonomy is also providing RPA in the form of software as a service (SaaS).

Technological disruptions and innovations have historically caused massive changes in corporations and industries, leading to industrial revolutions (Marengo, 2019). Currently, in the era of the Fourth Industrial Revolution, the widespread adoption of digital technology, AI, cloud services, and the internet of things are causing changes in industries, but technological innovations are not the only reason for introducing RPA. Japan is currently suffering from a low birthrate and is turning into an aging society that lacks human resources, leading to the "talent mismatch" phenomenon. These changes have led to a lack of skilled professionals and an excessive workload for office workers, necessitating a transformation from office workers into professionals; thus, RPA has been applied to replace work processes for which humans are not needed. This has reduced the amount of human resources needed for simple tasks, enabling the development of human resources centered on digital talents. This "talent-focused digital transformation" was planned for individuals, medium-sized and small businesses, the Japanese central government, other organizations, and educational institutions, facilitating the widespread application of RPA through open platforms.

Although corporations have paid considerable attention to RPA, there is a lack of academic research and case studies. The purpose of this case study is to provide insights and information about the development and application of RPA through cases at LG CNS and to suggest guidelines for corporations that adopt RPA in the future.

\section{Robotic Process Automation}

Robotic Process Automation (RPA) is a form of digital enablement technology that combines user interface (UI) and surface-level features to provide a graphical user interface (GUI) to automate tasks (Hofmann, Samp, \& Urbach, 2020). Automated routines for dedicated scripting languages and application programming interfaces were traditionally produced by software developers to automate tasks in the back-end system, whereas RPA systems leverage the UI and GUI, deploy predictive models for data transcription, and automate interactions. This allows easier application through existing systems and GUIs without the need for basic system changes or new development (Figure 1).

In 1980s, IT departments adopted enterprise resource planning tools to optimize and support management for innovation in task-related processes. This was followed by BPO as a cost-saving solution implemented by global IT corporations such as IBM and Oracle. The first product was released by the leading RPA corporation Blue Prism in 2003, followed by an automation library release by UiPath and Automation Anywhere. Starting in 2014, the term RPA arose from needs for software (robot)-based business process optimization, and RPA systems have been developed ever since. More recently, RPA has been developed into a "digital human resource," combining analytics and recognition technology to replace human tasks (Deloitte, 2018).

The Convergence Yoon (2017) defined six characteristics of RPA: agility, expandability, versatility, efficiency, safety, and accuracy (Table 1).
Development Tool

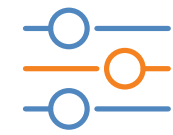

Process

Development Tool
Monitoring Server


Integrated Robot Management (Console)
Execution Robot
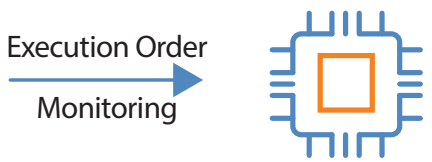

Automatic Execution Program (Software Worker)

Figure 1. Robotic process automation solution architecture. 
Table 1. Six characteristics of RPA (Robotic Process Automation)

\begin{tabular}{|c|c|}
\hline Characteristic & Description \\
\hline Agility & Quick set-up due to existing system-based implementation (approximately 1 week). \\
\hline Expandability/usability & No need for changes in existing information technology (IT) systems and new interface development. \\
\hline Versatility & Various systems, applications, communication methods, and interfaces. \\
\hline Efficiency & $\begin{array}{l}\text { Simple, repetitive tasks that previously required human resources are automatically executed, allowing more } \\
\text { efficient deployment of human resources. }\end{array}$ \\
\hline Safety & $\begin{array}{l}\text { Protection and security provided for internal, sensitive data from outside sources, with no need for additional } \\
\text { human resources. }\end{array}$ \\
\hline Accuracy & $\begin{array}{l}\text { Prevention of errors through input automation and reducing the need for repeated verification processes through } \\
\text { data integrity. }\end{array}$ \\
\hline
\end{tabular}

The IT consulting firm Gartner described the following quadrants for RPA companies: leaders, challengers, visionaries, and niche players. The corporations in the "magic quadrants" are evaluated along the axes of ability to execute and completeness of vision. Leaders in the market have an understanding of the realities of the market and have the capability to take opportunities and add additional functionality to products and services at the core of RPA services. Challengers in the market have a competitive ability to attract a large user following but are limited to a segment of the market. Visionaries are defined as innovators in the market for new emerging customer needs that offer new opportunities. Niche players specialize in functional or vertical areas with strong products that are specialized for specific subsets of the market or industry (Gartner Research, 2020).

The above findings show how a diverse range of corporations have different approaches and histories. According to the Gartner report, there was growth of over 50 times in slightly more than two years, and in this rapidly expanding market, there should be more competitors. Thus, startups and mega-corporations will appear and the delivery of RPA as a service will continue to develop through innovations.

\section{Case Analysis}

\section{Verification of External Information from Unassociated Systems}

Case 1. Verification of Accounts in Accounts Receivable at a US Corporation

The task was daily check of invoice status from customer client's website followed up by separate input of the invoices in AR Worksheet of ERP. The simple task started from browsing the website, logging in to Enterprise Portal, browsing the ERP AR, comparing Open AR list, and inputting Invoice status information. There was only one assigned worker, who had to complete 1,000 inputs per day. However, each task took 1 minute, leaving
$90 \%$ of the tasks incomplete daily. The implementation of RPA technology solved this problem by enabling all of these tasks to be completed, so that human resources could be deployed to focus on other human-needed tasks. This allowed the assigned worker to have more time for other tasks that involved decision-making, such as chargebacks and disputes. The receivables and bonds were fully updated, and the redeployment of human resources solved productivity issues.

\section{Case 2. Leased Department Sales Verification}

This case involved a simple task of extracting data on leased department sales and sending the data through email. Two collectors were assigned to this task, and there were 14 unassociated external websites. RPA was implemented to log into the partner portal websites, verify sales, download the sales data, carry out separate data processing for each department, and send the emails. RPA enabled 100\% automation of leased department sales verification. This reduced the number of tasks, allowing the human resources (i.e., the collectors) to focus on other tasks such as sales management.

\section{Simple Information Verification and System Input Task}

\section{Case 3. Purchase Bid Creation}

The simple task in this case was weekly purchase bid creation, in which the assigned worker created a parts code, followed by bid creation and execution. There were approximately 100 of these tasks per week (over 6,000 tasks yearly). Each task needed over 20 minutes (2,200 hours in total). RPA was implemented for the simple task of mapping and code creation through the SAP system. Over 6,000 of these tasks were automated, saving 2,216 work hours.

\section{Data Reconciliation}

\section{Case 4. Monitoring of Internal Control}

The purpose of this RPA implementation was to provide solu- 
tions for accounting firms in response to Internal Accounting Control System changes. RPA was implemented for wage verification, foreign currency conversion, and requests for unclaimed dividends. The automated tasks allowed full reconciliation of the data and preparation for inspection. It also acquired transparency and reduced the risk of verification errors (Cooper, Holderness, Sorensen, \& Wood, 2019).

\section{Intelligence Automation Roadmap}

\section{Case 5. AI-OCR Based Intelligent Automation}

Corporations that implement RPA often combine new digital innovations in each step of innovative task automation to augment the intelligence of RPA and expand the applicable task areas. In the 1990s and 2000s, RPAs were implemented through rule-based action tasks. As a development from basic automation, which automated simple macro-based tasks, early RPAs were applied to structured data input/output, external data collection (crawling), data input, and verification. These tasks were made possible by rule-engine, screen scraping, and workflow technologies. Currently, Intelligent Process Automation (IPA) supports decision-making through unstructured data analysis and learning. More recently, cognitive automation has been developed and implemented for process improvements and decision automation through technologies such as process mining/ task mining, text-to-speech, and predictive analytics, allowing the automation of data-based decision-making tasks (Figure 2).

This process has been facilitated by AI-driven improvements in optical character recognition (OCR) technology, allowing more solutions and accuracy through machine learning and deep learning (Figure 3).

LG CNS have implemented several RPAs to several tasks, however, is planning to develop, manage and provide AI service consultant in the future. Its plan to establish RPA management governance, RPA case sharing, framework developments, AI OCR implementation, text analysis, chatbot-linked services will be provided as LG CNS is planning to be a digital innovation leader in AI-RPA sector.

\section{Discussion}

In this era of rapid changes and the Fourth Industrial Revolution, RPA solutions are being developed and implemented in various industries and businesses. However, there are many considerations and concerns related to the implementation of RPA systems, as new technologies are being developed and agencies, companies, and markets are adapting to these changes.

(1) Application types and characteristics: Various tasks are automated by RPA; however, according to the Korean National Information Society Agency, RPA systems applied in public institutions can be divided into five types: external website
Rule-based Action taskoriented

\begin{tabular}{|l|}
\hline Basic Automation \\
\hline Macro-based limited \\
business automation \\
Applied work \\
- DB access and Data \\
Extraction. \\
- Single app, Macro app \\
Usage \\
Element technology \\
\hline - Macro and Script \\
\hline
\end{tabular}

\begin{tabular}{|c|}
\hline $\begin{array}{c}\text { RPA } \\
\text { (Robotic Process Automation) }\end{array}$ \\
\hline $\begin{array}{l}\text { Imitate user activities } \\
\text { based on structured rules }\end{array}$ \\
\hline Applied work \\
\hline $\begin{array}{l}\text { - Standardized Data } \\
\text { l/O between Systems } \\
\text { - External Data Collection } \\
\text { (Crawling) } \\
\text { - A Structured Data query } \\
\text { - Data Input and Verification }\end{array}$ \\
\hline Element technology \\
\hline $\begin{array}{l}\text { - Rule-Engine } \\
\text { - Screen Scraping } \\
\text {-Workflow }\end{array}$ \\
\hline
\end{tabular}

Expanding application to data-based decision-making tasks

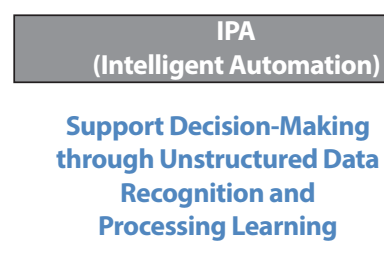

\section{Applied work}

- Extract/Input Text from Image Data

- Natural Language Query

- Generating Rules from Past Data

Element technology

- Al OCR
- Machine Visiion
- Chatbot
- Text Analysis: NLU, NLP, MRC

Cognitive Automation

Process Improvement, Decision-Making Automation

\section{Applied work}

- Automatic Discovery of

Automation Tasks

- Complicated Process

Improvement Tasks

- Predictive Decision-making

Element technology

- Process Mining/Task Mining

- NLG: Making Sentence

-TTS: Voice Talk

- Predictive Analytics

- ML/DL

Figure 2. Intelligent process automation roadmap. 


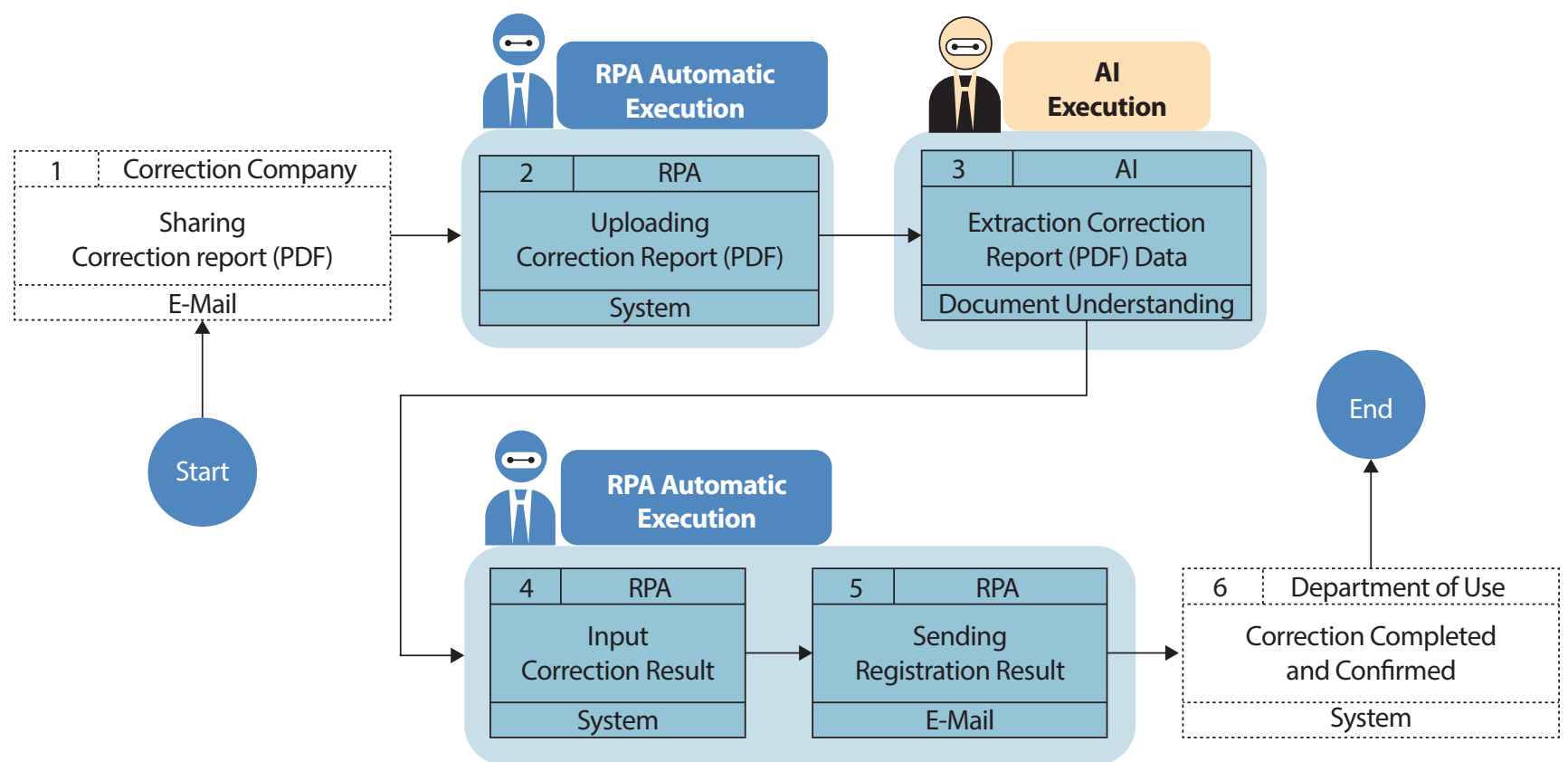

Figure 3. Al OCR application procedure. Al, artificial intelligence; OCR, optical character recognition.

information collection and input, structured report creation, simple repetitive internal system input, data comparison and verification, and automated email sending.

(2) Considerations: The main consideration for RPA implementation is to choose which processes to automate and how to do so. This choice can be made by setting automation goals for each department and identifying processes that can be automated with the highest efficiency and cost savings. The assigned workers' opinions should also be considered in this process.

When implementing an RPA, demonstration projects should be conducted to provide a proof of concept. Strategic governance should also be constructed for proper management through the Center of Excellence strategy, which sets the vision, goal, management, technological support, and standardization. The optimal automation solution should be chosen among various types of RPA solutions, using different natural languages. Whether a solution is optimized in terms of the organization, environment, and risk should also be reviewed before implementation. Data security measures are key for the stable execution of RPA, as access authority is crucial. Finally, legal and legislative measures should also be considered, as different policies apply to different industries or organizations. RPA might seem like a solution for all automatable tasks, but careless implementation will be ineffective and inefficient for the organization and impose financial/nonfinancial risks.

\section{Conclusion}

The automation of simple repetitive tasks is an unavoidable change as businesses and organizations work towards effectiveness and efficiency. Due to recent technological innovations, the combination of AI and RPA has enabled additional processes and functions such as unstructured data analysis. RPA has often been used in companies and private-sector organizations, but it has also been recently implemented in the public sector. However, the implementation of RPA is not always the correct solution, and its efficiency, convenience, and financial aspects should be carefully considered. As the RPA market is growing rapidly, further research and case studies are required for future applications of RPA.

\section{Acknowledgements}

This work was supported by the Ministry of Education of the Republic of Korea and the National Research Foundation of Korea (NRF-2019S1A3A2098438).

\section{References}

Anagnoste, S. (2017). Robotic Automation Process: The next major revolution in terms of back office operations improvement. Proceedings of the International Conference on Business Excellence (vol. 11, no. 1, pp. 676-686). Bucharest, Romania. 
Cooper, L. A., Holderness, D. K. Jr, Sorensen, T. L., \& Wood, D. A. (2019). Robotic process automation in public accounting. Accounting Horizons, 33(4), 15-35.

Deloitte, L. L. P. (2018). The robots are waiting: Are you ready to reap the benefits? London, UK: Deloitte.

Devarajan, Y. (2018). A study of robotic process automation use cases today for tomorrow's business. International Journal of Computer Techniques, 5(6), 12-18.

Gartner Research. (2020, July 27). Gartner magic quadrant for robotic process automation. Retrieved from https://www.gartner.com/ en/documents/3988021/magic-quadrant-for-robotic-processautomation
Hofmann, P., Samp, C., \& Urbach, N. (2020). Robotic process automation. Electronic Markets, 30(1), 99-106.

Marengo, L. (2019). Is this time different? A note on automation and labour in the fourth industrial revolution. Journal of Industrial and Business Economics, 46(3), 323-331.

Syed, R., Suriadi, S., Adams, M., Bandara, W., Leemans, S. J., Ouyang, C., ... Reijers, H. A. (2020). Robotic process automation: Contemporary themes and challenges. Computers in Industry, 115, 103162.

Yoon, I. Y. (2017). Fusion of robot and business, business process automation. Seoul, Korea: Convergence Research Policy Center. 